\title{
SET QUANTITIES AND TAUBERIAN OPERATORS
}

\author{
SERGIO FALCON AND KISHIN SADARANGANI
}

Received 6 July 2001

The concept of convexity plays an important role in the classical geometry of normed spaces and it is frequently used in several branches of nonlinear analysis. In recent years some papers that contain generalizations of the concept of convexity with the help of the measures of noncompactness have appeared. The Tauberian operators were introduced by Kalton and Wilansky (1976) and they appear in the literature with the aim of responding to some questions related with the summability and the factorization of operators; in the preservation by isomorphisms in Banach spaces, and so forth. In this paper we make the study of the Tauberian operators, not starting from the Euclidean distance, but by means of general set quantities.

\section{Introduction}

The concept of convexity plays an important role in the classical geometry of normed spaces. It is frequently used in the metric fixed point theory and other branches of nonlinear analysis [6, 8, 9, 11, 13].

In recent years there have appeared some papers that contain generalizations of the concept of convexity with the help of the measures of noncompactness [3, 12, 13]. Recently, Cabrera [7] has introduced the concept of weak near uniform convexity using the De Blasi measure of weak noncompactness [10].

In order to recall this concept we introduce some notation. Assume that $(E,\|\cdot\|)$ is an infinite-dimensional Banach space with the zero element $\theta$. Denote by $B_{E}$ and $S_{E}$ the closed unit ball and the unit sphere in $E$, respectively. For a subset $X$ of $E$ we denote by conv $X$ the convex hull and by Conv $X$ the convex closed hull of $X$.

Moreover, if we assume that $X$ is a nonempty and bounded set in $E$ then the quantity $\chi(X)$ defined in the following way: $\chi(X)=\inf \{\epsilon>0$ : there exists 
a compact set $Y$ such that $\left.X \subset Y+\epsilon B_{E}\right\}$ is called the Hausdorff measure of noncompactness of the set $X$.

For the properties of the function $\chi$ we refer to [4]. We recall the concept of the De Blasi measure of weak noncompactness.

Let $X$ be a nonempty and bounded set in $E$. The quantity $\beta(X)$ defined in the following way: $\beta(X)=\inf \{\epsilon>0$ : there is a weakly compact set $Y$ such that $\left.X \subset Y+\epsilon B_{E}\right\}$ is called the De Blasi measure of weak noncompactness in the space $E$. Observe that in the case when $E$ is reflexive, we have that $\beta(X)=0$ for every nonempty and bounded set $X$ in $E$. We refer to [5] for more details.

We only mention that $\beta(X) \leq \chi(X)$ for any nonempty and bounded set $X$ in $E$. The family of all nonempty and bounded subsets of $E$ will be denoted by $P_{b}(E)$. For $C, D \in P_{b}(E)$ we consider the Hausdorff nonsymmetric distance defined in the following way:

$$
H^{\prime}(C, D)=\inf \{\epsilon>0: C \subset B(D, \epsilon)\}=\inf \{\epsilon>0: C \subset D+\epsilon B\} .
$$

The Hausdorff distance between $C$ and $D$ is defined as

$$
H(C, D)=\max \left\{H^{\prime}(C, D), H^{\prime}(D, C)\right\} .
$$

Observe the function $H$ is a metric on the family $P_{b c}(E)$ where

$$
P_{b c}(E)=\left\{C \in P_{b}(E): C=\bar{C}\right\} .
$$

In what follows, let $\mathcal{N}$ be a nonempty subfamily of $P_{b}(E)$. Consider two real functions defined on the family $P_{b}(E)$ in the following way:

$$
\begin{aligned}
& H_{\mathcal{N}}^{\prime}(C)=\inf \left\{H^{\prime}(C, P): P \in \mathcal{N}\right\}, \\
& H_{\mathcal{N}}(C)=\inf \{H(C, P): P \in \mathcal{N}\} .
\end{aligned}
$$

For further goals we recall the function $H_{\mathcal{N}}$ was considered in [5] on a complete metric space $S$. The main result which appears in [5] is formulated in the following proposition.

Proposition 1.1. Let $N$ be a nonempty subfamily of $P_{b}(S)$ satisfying the condition

$$
M \in \mathcal{N}, \quad \emptyset \neq P \subset M \Longrightarrow P \in \mathcal{N} .
$$

Then for every $C \in P_{b}(S)$ the following equality holds:

$$
H_{\mathcal{N}}^{\prime}(C)=H_{\mathcal{N}}(C) .
$$

If $\mathcal{N}$ is the family of all nonempty and relatively compact subsets of $E$, then $H_{\mathcal{N}}$ coincides with the measure of noncompactness of Hausdorff, $\chi$.

And if $\mathcal{N}$ is the family of all nonempty and relatively weakly compact subsets of $E$, then $H_{\mathcal{N}}$ coincides with the measure of weak noncompactness of De Blasi, $\beta$. 
Definition 1.2. A mapping $\mu: P_{b}(E) \rightarrow[0, \infty)$ is said to be a set quantity if it satisfies the following conditions for $C, D \in P_{b}(E)$ and $\lambda \in \mathscr{R}$ :

(1) $\mu(C \cup D)=\max \{\mu(C), \mu(D)\}$

(2) $\mu(\lambda C)=|\lambda| \mu(C)$

(3) $\mu(C+D) \leq \mu(C)+\mu(D)$

(4) $\mu(\operatorname{conv} C)=\mu(C)$.

From this definition it is easily seen that $\mu$ satisfies:

(1) $\mu(\{0\})=0$

(2) $C \subset D \Rightarrow \mu(C) \leq \mu(D)$

(3) $\mu(C)=\mu(\bar{C})$.

The family $\operatorname{ker} \mu$ is defined in the usual way as $\operatorname{ker} \mu=\left\{C \in P_{b}(E)\right.$ : $\mu(C)=0\}$ and will be called the kernel of the quantity $\mu$.

When $\operatorname{ker} \mu$ is the collection of all nonempty and relatively compact subsets of $E$ then $\mu$ is referred to as a measure of noncompactness. In the case when $\operatorname{ker} \mu$ is the family of all nonempty and relatively weakly compact subsets of $E$ we will say that $\mu$ is a measure of weak noncompactness. So, $\chi$ and $\beta$ are two particular examples of set quantities.

Definition 1.3. A Banach space $E$ is $\mu$-uniformly convex ( $\mu$-UC, in short) if

$$
\lim _{\epsilon \rightarrow 0}\left\{\sup \left\{\mu(F(f, \epsilon)): f \in S_{E^{*}}\right\}\right\}=0
$$

where $\mu$ is a set quantity and $F(f, \epsilon)=\left\{x \in B_{E}: f(x) \geq 1-\epsilon\right\}$.

Similarly we define the next concept.

Definition 1.4. A space $E$ is said to be $\mu$-locally uniformly convex ( $\mu$-LUC, in short) if

$$
\lim _{\epsilon \rightarrow 0}\left\{\mu(F(f, \epsilon)): f \in S_{E^{*}}\right\}=0
$$

Now, we give the following definition.

Definition 1.5. A space $E$ is referred to as $\mu$-strictly convex ( $\mu$-SC, in short) if $\mu(F(f, 0))=0$ for each $f \in S_{E^{*}}$ such that $F(f, 0) \neq \emptyset$.

Notice that $\mu$-UC $\Rightarrow \mu$-LUC $\Rightarrow \mu$-SC.

\section{2. $\mu$-Tauberian operators}

The Tauberian operators were introduced by Kalton and Wilansky in [15] and they appear in the literature with the objective of responding to a group 
of questions related with the addition [18] and the factorization of operators [9]; in the preservation for isomorphisms in Banach spaces, and so forth. In [15, Theorem 2.2] they characterized the Tauberian operators in the following way and this will be our starting point: An operator $T \in \mathscr{L}(E, F)$ is said to be Tauberian and we will denote it by $T \in \tau(E, F)$, if for any $C \in P_{b}(E)$ with $T(C)$ relatively weakly compact, one has that $C$ is relatively weakly compact.

Next we generalize this definition with the help of a set quantity $\mu$ and we will obtain the $\mu$-Tauberian operators.

More concretely, one has the following definition.

Definition 2.1. An operator $T \in \mathscr{L}(E, F)$ is said to be $\mu$-Tauberian, where $\mu$ is a set quantity, and we will denote it by $T \in \tau_{\mu}(E, F)$, if for any $C \in P_{b}(E)$ with $T(C)$ relatively weakly compact one has that $C \in \operatorname{Ker} \mu$.

In the following proposition we will see how weakly compact disturbs preserve the $\mu$-Tauberian operators, in the same way that in the standard case of Tauberian operators.

Proposition 2.2. Let $T$ be a $\mu$-Tauberian operator from $E$ into $F$ and $K a$ weakly compact operator. Then $T+K \in \tau_{\mu}(E, F)$.

Proof. Let $C$ be a nonempty and bounded set of $E$ and consider that $(T+K)(C)$ is relatively weakly compact. As $K$ is a weakly compact operator, $K(C)$ will be relatively weakly compact and as $T(C) \subset(T+K)(C)-K(C)$, keeping in mind the properties of the De Blasi measure of weak noncompactness, one will have that $\beta(T(C)) \leq \beta((T+K)(C))+\beta(K(C))=0$ and consequently, $T(C)$ is relatively weakly compact. Taking into account that $T \in \tau_{\mu}(E, F)$, then $C \in \operatorname{Ker} \mu$. This completes the proof.

One of the most interesting properties in the Tauberian operators is that their kernels are reflexive. We show some relationships with this fact.

In [7], it is proved that for the set quantity $\beta$, De Blasi measure of weak noncompactness, the class of the $\beta$-UC spaces and the class of the $\beta$-LUC spaces coincide with the class of the reflexive ones. Take into account that the class of the reflexive spaces in fact coincides with the class of the Banach spaces for those that the De Blasi measure of weak noncompactness is null.

On the other hand, to keep in mind that set quantities exist for which Banach spaces of infinite dimension do not exist in those that this quantity is null. Take as example the set quantity $\chi$, the Hausdorff measure of noncompactness that is only null in the finite-dimensional spaces. And more ahead we will see that the fact that a set quantity is not null in infinite-dimensional Banach spaces will be fundamental for our development. 
Recapturing the question that concerns us again and keeping in mind what was said previously, the property that the kernels of Tauberian operators are reflexive that is, if $T$ is a Tauberian operator from $E$ into $F$, then its kernel $\operatorname{Ker} T$ is a space for which the quantity $\beta$ is null. This means, in particular that $\operatorname{Ker} T$ is a $\beta$-LUC space.

THEOREM 2.3. If $T$ is a $\mu$-Tauberian operator from $E$ into $F$, then $\operatorname{Ker} T$ is a Banach space for which the quantity $\mu$ is null and, therefore, it is a $\mu$-UC space.

Proof. Suppose that $T \in \tau_{\mu}(E, F)$ and let $A$ be a nonempty and bounded set of the kernel of $T$. As $A \subset \operatorname{Ker} T$, then $T(A)=0$, that is to say that $T(A)$ is relatively weakly compact. As $T$ is $\mu$-Tauberian, $A \in \operatorname{Ker} \mu$, that is, $\mu(A)=0$. Therefore, the quantity $\mu$ is null in $\operatorname{Ker} T$. This completes the proof.

This theorem already has as corollary the result acquaintance for the Tauberian operators and mentioned previously.

Corollary 2.4. If $T$ is a $\beta$-Tauberian operator, that is to say, if $T \in \tau_{\beta}(E, F)$, then $\operatorname{Ker} T$ is reflexive.

The test is immediate, because it is enough to keep in mind that the spaces for those that the De Blasi measure of weak noncompactness is null they are the reflexive ones.

To notice that if $\mu$ is a set quantity for which Banach spaces of infinite dimension in those it is not null do not exist, we have the following corollary to Theorem 2.3.

COROLlaRY 2.5. Let $\mu$ be a set quantity for which there are no Banach spaces of infinite dimension in those it is null and let $T$ be a $\mu$-Tauberian operator from $E$ into $F$. Then $\operatorname{Ker} T$ is of finite dimension.

In particular, as the Hausdorff measure of noncompactness $\chi$ verifies the hypothesis of the preceding corollary, we have the following corollary.

Corollary 2.6. Let $T$ be a $\chi$-Tauberian operator from $E$ into $F$. Then $\operatorname{Ker} T$ is finite dimensional.

For the following result, we will demand some additional condition to the set quantity $\mu$.

This way, we will give the following necessary definition to continue this development. 
Definition 2.7. Let $E$ be a set and $\mathscr{A}$ a family of subsets of $E$. We will say that $\mathscr{A}$ is sequentially determined, if for each $A \subset E$, it is verified that $A \in \mathscr{A}$ if and only if for any sequence $\left(x_{n}\right) \subset A$, the set $\left\{x_{n}: n \in N\right\} \in \mathscr{A}$.

Examples of sequentially determined families are that of the relatively compact sets and that of the relatively weakly compact ones of a Banach space $E$.

Definition 2.8. Let $T$ be a linear and continuous operator between two Banach spaces $E$ and $F$, that is to say, $T \in \mathscr{L}(E, F)$. We define the $\mu$-variation of $T$ and we indicate it as $\mu(T)$, as the number $\mu(T)=\mu\left(T\left(B_{E}\right)\right)$.

With the following result we will obtain an algebraic characterization of the $\mu$-Tauberian operators.

THEOREM 2.9. Let $\mu$ be a set quantity for which $\operatorname{Ker} \mu$ is sequentially determined. Then, an operator $T: E \rightarrow F$ is $\mu$-Tauberian if and only if given a Banach space $G$ and an operator $L: G \rightarrow E$ such that $T \circ L$ is weakly compact, then $L$ has null $\mu$-variation.

Proof. Suppose that $T: E \rightarrow F$ is $\mu$-Tauberian and let $L: G \rightarrow E$ be an operator such that $T \circ L$ is weakly compact. By virtue of the weak compactness of $T \circ L$ one has that $(T \circ L)\left(B_{G}\right)$ is relatively weakly compact, or what is the same thing, $T\left(L\left(B_{G}\right)\right)$ is relatively weakly compact. As $T$ is $\mu$ Tauberian, $L\left(B_{G}\right) \in \operatorname{Ker} \mu$ and, as a consequence, $L$ has null $\mu$-variation, as required.

Suppose that $T: E \rightarrow F$ verifies that for each Banach space $G$ and an operator $L: G \rightarrow X$ such that $T \circ L$ is weakly compact, then $L$ has null $\mu$-variation. If $T$ is not $\mu$-Tauberian, this means that $A \in P_{b}(E)$ exists, with $T(A)$ relatively weakly compact and $A \notin \operatorname{Ker} \mu$.

As the set quantity $\mu$ verifies that its kernel $\operatorname{Ker} \mu$ is sequentially determined then there exists $\left(x_{n}\right) \subset A$ and $\left\{x_{n}: n \in N\right\} \notin \operatorname{Ker} \mu$.

Consider the operator $L: l^{1} \rightarrow X: L\left(e_{n}\right)=x_{n}$. Then, as $(T \circ L)\left(e_{n}\right) \subset T(A)$, $(T \circ L)\left(B_{1}^{1}\right) \subset$ a $\operatorname{Conv}(T(A))$, where a Conv $X$ denotes the absolutely convex hull of $T(A)$ that is relatively weakly compact because $T(A)$ is. Therefore, $T \circ L$ is weakly compact and, from our hypothesis, $\mu\left(L\left(B_{1}^{1}\right)\right)=0$.

On the other hand, as $\left\{x_{n}: n \in N\right\} \subset L\left(B_{1}^{1}\right)$ and $\left\{x_{n}: n \in N\right\} \notin \operatorname{Ker} \mu$ it will be that $\mu\left(L\left(B_{1}^{1}\right)\right)>0$, which is absurd.

In particular, as the Tauberian operators are in fact the $\beta$-Tauberian, and as the family of the relatively weakly compact ones is sequentially determined, one can obtain the following result as a corollary of this theorem and that appeared in [11, Corollary 1.6]. 
Corollary 2.10. An operator $T: E \rightarrow F$ is Tauberian if and only if given a Banach space $G$ and an operator $L: G \rightarrow E$ that it verifies that $T \circ L$ is weakly compact, then $T$ is weakly compact.

Maybe one of the main reasons for the study of the Tauberian operators was the inheritance of isomorphic properties of the Banach spaces. This way, we have that if $T: E \rightarrow F$ is a Tauberian operator and $F$ has the property of reflexivity (quasi-reflexivity, weak sequentially complete, etc.), then $E$ also possesses it.

In our context of $\mu$-Tauberian operators we have, in certain measure, some homologous properties and, for it, it is necessary to keep in mind that the reflexive spaces are those for which the De Blasi measure is null. This way, we have the following result.

THEOREM 2.11. If $T: E \rightarrow F$ is $\mu$-Tauberian and if $F$ is reflexive, then the set quantity $\mu$ is null on $E$.

Proof. Let $A$ be a nonempty and bounded set of $E$. Then, as $F$ is reflexive, $T(A)$ will be, when being bounded, relatively weakly compact. Taking into acount that $T$ is $\mu$-Tauberian, it will be that $A \in \operatorname{Ker} \mu$. Consequently, $\mu$ is null in $E$, like we wanted to prove.

In particular, if we apply this theorem to the set quantity $\beta$, we already obtain the well-known result of the classic Tauberian operators. More concretely, one has the following result.

Corollary 2.12. If there exists $T: E \rightarrow F$ Tauberian and $F$ is reflexive, then $E$ is reflexive.

Take into account that if for the set quantity $\mu$ there are no infinite-dimensional spaces for which $\mu$ is null. Suppose that $\mu$ is only null in finite-dimensional spaces, Theorem 2.11 allows us to obtain the following corollary.

Corollary 2.13. Let $E$ and $F$ be real Banach spaces of dimension infinity and suppose that $\mu$ is not null in infinite-dimensional spaces and that $F$ is reflexive. Then, $\mu$-Tauberian operators do not exist from $E$ into $F$. That is to say, $\tau_{\mu}(E, F)=\emptyset$.

In particular, if we take the Hausdorff measure of noncompactness, one has the following corollary.

Corollary 2.14. If $F$ is reflexive, then $\tau_{\chi}(E, F)=\emptyset$. 
Under the hypothesis that the relatively weakly compact sets are contained in $\operatorname{Ker} \mu$, one has the following corollary.

Corollary 2.15. If $P_{\mathrm{rwc}}(E) \subset \operatorname{Ker} \mu$, then $I_{E} \in \tau_{\mu}(E, E)$, being $P_{\mathrm{rwc}}(E)$ the family of the relatively weakly compact subsets of $E$.

Also, for Theorem 2.11, we have the following consequence.

Corollary 2.16. If $E$ is a Banach space for which the set quantity $\mu$ is not null, then any $f \in E^{*}$ is not $\mu$-Tauberian.

As an example, we will study the set $\tau_{\mu}\left(l^{\infty}, c_{0}\right)$, but before we will recall the following theorem of James [14]:

$A$ Banach space $E$ is reflexive if and only if for each $f \in E^{*}-\{0\}$ there is $x \in E-\{0\}$ so that $f(x)=\|f\| \cdot\|x\|$.

Definition 2.17. A set quantity $\mu$ defined on a Banach space $E$ is said to be cantorian if for any decreasing sequence $\left(A_{n}\right)$ of nonempty sets of $E$, bounded and closed so that $\lim _{n \rightarrow \infty} \mu\left(A_{n}\right)=0$ then $\bigcap_{1}^{\infty} A_{n} \neq \emptyset$.

In [17], it is proven that all operators $T: L^{\infty} \rightarrow c_{0}$ are weakly compact. Taking this fact into account, we will prove the following result.

THEOREM 2.18. If $\mu$ is a cantorian set quantity, then $\tau_{\mu}\left(l^{\infty}, c_{0}\right)=\emptyset$.

Proof. Suppose that $T \in \tau_{\mu}\left(l^{\infty}, c_{0}\right)$. For the result mentioned above, $T$ is weakly compact, that is, for any $A \in P_{b}\left(l^{\infty}\right), T(A)$ is relatively weakly compact. As $T$ is $\mu$-Tauberian, $A \in \operatorname{Ker} \mu$ and, as a consequence, the set quantity $\mu$ is null on $l^{\infty}$. Therefore, in particular, $l^{\infty}$ is $\mu$-LUC.

Taking into account the theorem of James, like $\mu$ is a cantorian set quantity, it would be that $l^{\infty}$ is reflexive, which is absurd. Therefore $\tau_{\mu}\left(l^{\infty}, c_{0}\right)=\emptyset$.

Theorem 2.18 generalizes the well-known result that there is no Tauberian operator from $l^{\infty}$ into $c_{0}$, because the De Blasi measure of weak noncompactness is cantorian.

More concretely, we have the following corollary.

Corollary 2.19. There is no Tauberian operator from $l^{\infty}$ into $c_{0}$.

A fact also fundamental in the theory of Tauberian operators is that if $T^{* *}$ is Tauberian, then $T$ is Tauberian, but the converse implication is not verified [1]. We will see that this result is also completed in the context of the $\mu$-Tauberian operators, although with certain restrictions for the set quantity $\mu$. 
Definition 2.20. A set quantity $\mu$ is said to be equivalent by isometries if for any isometry $I: E \rightarrow F$ there are two constants $k$ and $k^{\prime}$ that depend on the isometry so that for any $C \in P_{b}(E)$ it is verified that $k \mu(C) \leq \mu(I(C)) \leq k^{\prime} \mu(C)$.

THEOREM 2.21. Suppose that the set quantity $\mu$ is equivalent for isometries. If $T^{* *}$ is $\mu$-Tauberian, then $T$ is $\mu$-Tauberian.

Proof. Consider the following commutative diagram:

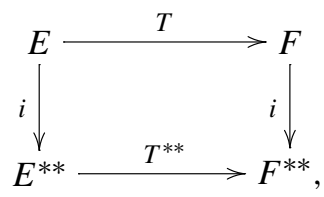

where $i$ is the canonical map from a space into its bidual and suppose $T^{* *}$ is Tauberian.

Let $A \in P_{b}(E)$ and $T(A)$ be relatively weakly compact. As every linear and continuous operator transforms relatively weakly compact sets into relatively weakly compact sets, then $i(T(A))$ is relatively weakly compact. By virtue of the commutative law of the previous diagram, $T^{* *}(i(A))$ is relatively weakly compact. As $T^{* *}$ is $\mu$-Tauberian, then $i(A) \in \operatorname{Ker} \mu$. Taking into account the hypothesis that the quantity $\mu$ is equivalent for isometries, then $A \in \operatorname{Ker} \mu$ and, as a consequence, $T$ is $\mu$-Tauberian.

It is necessary to notice that the result for the classic Tauberian operators, one cannot deduce as a corollary of Theorem 2.21, since as Astala and Tylli proved [2], the De Blasi measure of weak noncompactness is not equivalent for isometries. Nevertheless, to notice that in Theorem 2.21, the essential thing is that the $\operatorname{Ker} a$ is preserved by isometries, or in equivalent form, $\mu \in \operatorname{Ker} \mu \Leftrightarrow$ $I(A) \in \operatorname{Ker} \mu$ for any isometry $I$. Thus, this would be the enough hypothesis for Theorem 2.21. This hypothesis is verified for the De Blasi measure of weak noncompactness, by virtue of the call approach of Eberdin [16, page 159].

\section{References}

[1] T. Alvarez and M. Gonzalez, Some examples of Tauberian operators, Extracta Math. 5 (1990), no. 3, 91-107.

[2] K. Astala and H.-O. Tylli, Seminorms related to weak compactness and to Tauberian operators, Math. Proc. Cambridge Philos. Soc. 107 (1990), no. 2, 367-375. MR 91b:47016. Zbl 0709.47009.

[3] J. Banas, On drop property and nearly uniformly smooth Banach spaces, Nonlinear Anal. 14 (1990), no. 11, 927-933. MR 92f:46011. Zbl 0734.46005.

[4] Compactness conditions in the geometric theory of Banach spaces, Nonlinear Anal. 16 (1991), no. 7-8, 669-682. MR 92b:46016. Zbl 0724.46019. 
[5] J. Banas and K. Fraczek, Conditions involving compactness in geometry of Banach spaces, Nonlinear Anal. 20 (1993), no. 10, 1217-1230. MR 94h:46020. Zbl 0821.46010.

[6] B. Beauzamy, Introduction to Banach Spaces and Their Geometry, North-Holland Mathematics Studies, vol. 68, North-Holland, Amsterdam, 1982. MR 84g:46017. Zbl 0491.46014.

[7] I. Cabrera, Medidas de no compacidad débil y geomrtría de espacios de Banach, Ph.D. thesis, Universidad de Las Palmas de Gran Canaria, 1999.

[8] J. A. Clarkson, Uniformly convex spaces, Trans. Amer. Math. Soc. 40 (1936), no. 3, 396-414. CMP 1501 880. Zbl 0015.35604.

[9] W. J. Davis, T. Figiel, W. B. Johnson, and A. Pelczynski, Factoring weakly compact operators, J. Funct. Anal. 17 (1974), 311-327. MR 50\#8010. Zbl 0306.46020.

[10] F. S. De Blasi, On a property of the unit sphere in a Banach space, Bull. Math. Soc. Sci. Math. R. S. Roumanie (N.S.) 21(69) (1977), no. 3-4, 259-262. MR 58\#2475. Zbl 0365.46015.

[11] K. Goebel and W. A. Kirk, Topics in Metric Fixed Point Theory, Cambridge Studies in Advanced Mathematics, vol. 28, Cambridge University Press, Cambridge, 1990. MR 92c:47070. Zbl 0708.47031.

[12] K. Goebel and T. Sekowski, The modulus of noncompact convexity, Ann. Univ. Mariae Curie-Skłodowska Sect. A 38 (1984), 41-48 (1986). MR 87j:46031. Zbl 0607.46011.

[13] R. Huff, Banach spaces which are nearly uniformly convex, Rocky Mountain J. Math. 10 (1980), no. 4, 743-749. MR 82b:46016. Zbl 0505.46011.

[14] R. C. James, Reflexivity and the supremum of linear functionals, Israel, J. Math. 13 (1972), 289-300.

[15] N. Kalton and A. Wilansky, Tauberian Operators on Banach Spaces, Proc. Amer. Math. Soc. 57 (1976), no. 2, 251-255. MR 57\#13555. Zbl 0304.47023.

[16] V. Lakshmikantham and S. Leela, Nonlinear Differential Equations in Abstract Spaces, International Series in Nonlinear Mathematics: Theory, Methods and Applications, vol. 2, Pergamon Press, Oxford, 1981. MR 82i:34072. Zbl 0456.34002.

[17] J. Lindenstrauss, On the modulus of smoothness and divergent series in Banach spaces, Michigan Math. J. 10 (1963), 241-252. MR 29\#6316. Zbl 0115.10001.

[18] R. D. Neidinger, Properties of Tauberian operators on Banach spaces, Ph.D. thesis, University of Texas and Austin, 1984.

Sergio Falcon: Department of Mathematics, University of Las Palmas de Gran Canaria, 35017 Las Palmas, Spain

E-mail address: sfalcon@dma. ulpgc. es

Kishin Sadarangani: Department of Mathematics, University of Las Palmas de Gran Canaria, 35017 Las Palmas, Spain 


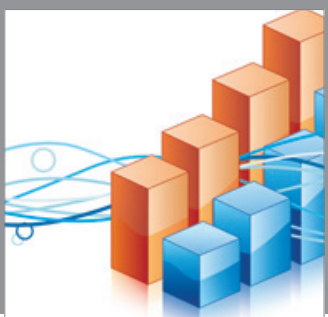

Advances in

Operations Research

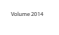

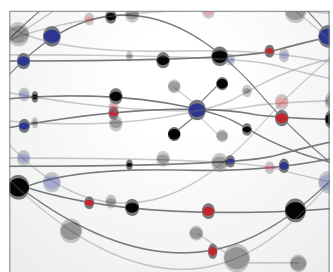

\section{The Scientific} World Journal
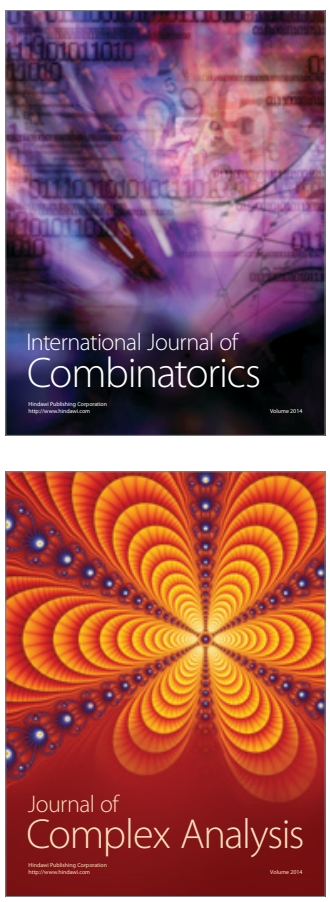

International Journal of

Mathematics and

Mathematical

Sciences
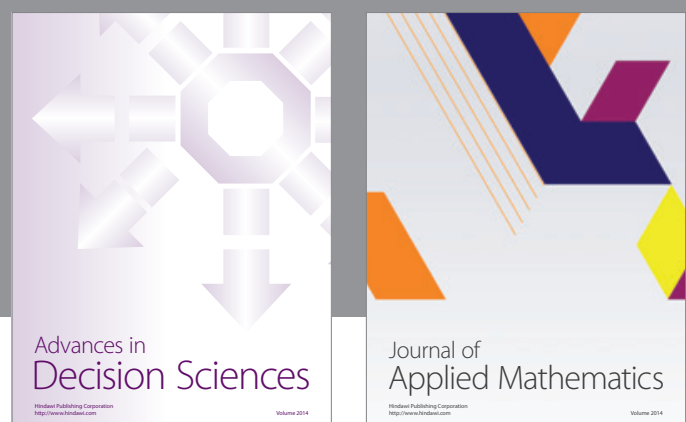

Journal of

Applied Mathematics
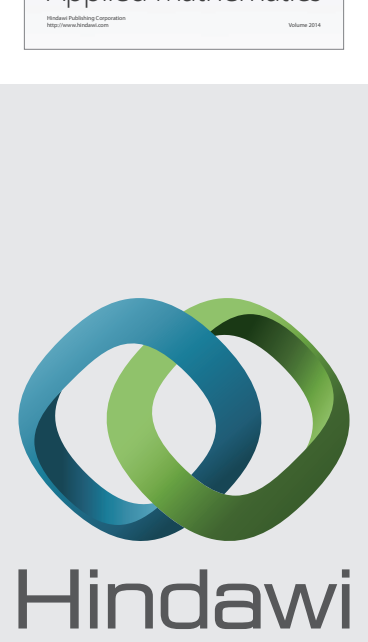

Submit your manuscripts at http://www.hindawi.com
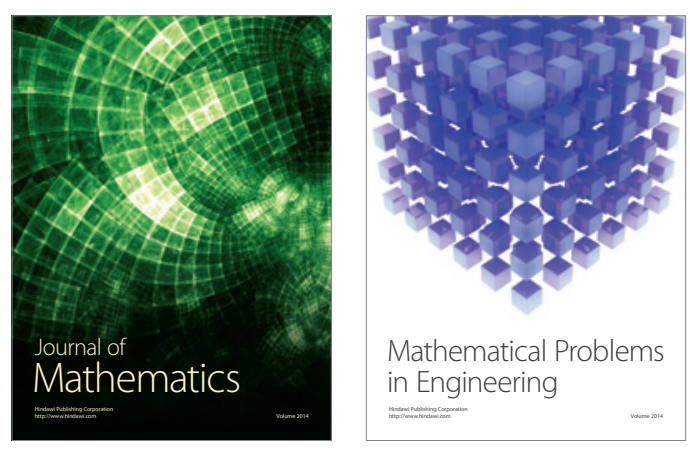

Mathematical Problems in Engineering
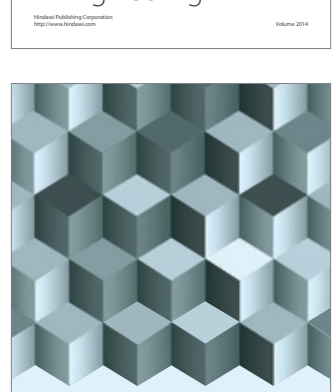

Journal of

Function Spaces
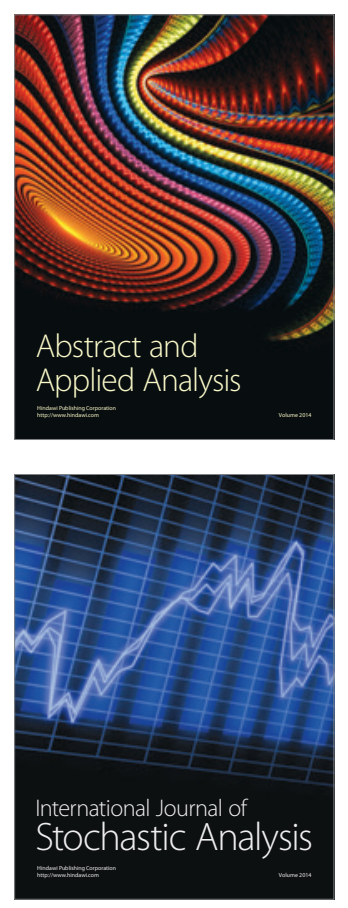

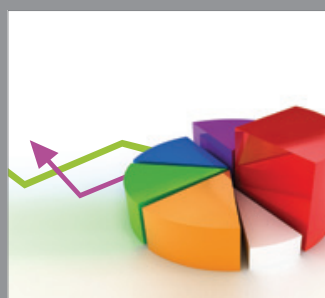

ournal of

Probability and Statistics

Promensencen
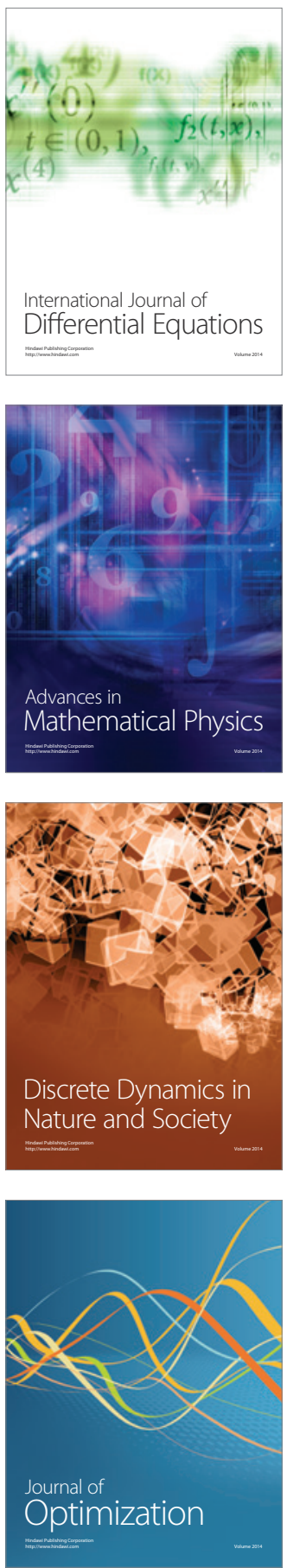